\title{
Research and Exploration of Pharmaceutical Analysis MES Module Reform in Vocational Colleges Pharmaceutical Quality \& Safety Speciality+
}

\author{
Wang Wenbin*, Chen Benshou*, Liu Zhonghong, Cao Chunmei, Li Pengxi, Ren Xiaoli \\ Environmental and Quality Testing Department, Chongqing Chemical Industry Vocational College, Chongqing, China
}

Email address:

wwb601@126.com (Wang Wenbin), cbs7575@sina.com (Chen Benshou)

*Corresponding author

To cite this article:

Wang Wenbin, Chen Benshou, Liu Zhonghong, Cao Chunmei, Li Pengxi, Ren Xiaoli. Research and Exploration of Pharmaceutical Analysis MES Module Reform in Vocational Colleges Pharmaceutical Quality \& Safety Speciality+. Science Journal of Education.

Vol. 6, No. 4, 2018, pp. 110-115. doi: 10.11648/j.sjedu.20180604.11

Received: August 28, 2018; Accepted: September 19, 2018; Published: October 11, 2018

\begin{abstract}
From the perspective of the supply side of higher vocational education, this paper has carried out the research and exploration of more than four years of MES module reform on Pharmaceutical analysis, the core course of drug quality and safety. The module design of "Pharmaceutical analysis" MES module reform is described. The concrete measures of the reform include the optimization of teaching content, the establishment and operation of the organization and implementation of the teaching organization, the integration of PBL teaching mode in the teaching model and the innovation of the examination methods. It also summarizes some problems arising from the reform process and the corresponding measures for these problems. The reform takes the drug food safety as the guidance, clear the importance of the Pharmaceutical Analysis knowledge to ensure the safety of drug use, based on the typical Pharmacopoeia analysis method, learning the new technology of Pharmaceutical Analysis experiment in the use of drug testing, paying attention to the teaching methods and methods, transforming the abstract into intuitionistic and reducing the difficulty of the theory of the theory. The reform measures of Pharmaceutical Analysis MES enlighten students' interest in learning better, and make students realize that drug quality testing is a guarantee subject closely related to the healthy development of the pharmaceutical industry; the introduction of the research frontier of Pharmaceutical Analysis and the introduction of typical methods and techniques makes students in the actual work in the future and develop drug quality inspection, the new idea of measuring technology.
\end{abstract}

Keywords: Pharmaceutical Quality \& Safety Speciality, Pharmaceutical Analysis, MES Module, Reform PBL Teaching Mode

\section{Introduction}

The reform of the supply side of higher vocational education is to improve the quality, efficiency and creativity of the supply side of the education, and make it close to the consumption needs and consumption habits of the students so as to fulfill the needs of individual development and the needs of the future society. The supply structure of higher vocational education provides students with new rich and diverse educational resources, educational environment and service mode, breaking the original structure, replacing a single training mode, unifying the curriculum resources, strict assessment and evaluation of the supply structure. [1] On the other hand, it enriches the supply structure of education, provides students with rich, multiple, optional educational resources, educational environment and the new supply side structure of education service mode, replacing and breaking the original single training mode, unified curriculum resources and rigid examination evaluation supply structure. The core element of education is not to be good at processing, but is good at discovering and discovering the endowment of every child and further protecting and supporting their growth, which is the attribute of education [2].

According to the characteristics of different majors, Song Lili and others introduced comprehensive experiments into the drug analysis course experiment, combined with the actual 
situation of the school, combined with the job and work process, combined with production practice, to guide students to carry out independent experiments [3-6]. Xiong Yan and others, based on the background of the application-oriented development of Applied Colleges and universities, carried out a hierarchical, modular personnel training model reform in view of the existing problems of drug analysis curriculum. In terms of teaching methods [7-11]. Fu Chunyan [12] and others discussed the application of inquiry teaching method in pharmaceutical analysis teaching experiment through teaching cases. Wu Lingfeng [13, 14] and others introduced PBL teaching method in the course of drug analysis of clinical specialty, which stimulated the interest and enthusiasm of students in this course.

The Pharmaceutical Quality \& Safety Speciality of Chongqing Chemical Industry Vocational College (CCIVC) is one of the most major students in our college, and Pharmaceutical Analysis is one of the core courses of the major. The modular reform test class was first set up among the 2014 class students, which opened the prelude to the modulus reform of the drug quality and safety major and the Pharmaceutical Analysis course MES. After more than three years of research and exploration, some useful experiences and shortcomings were obtained. From the perspective of the supply side, the MES module reform focuses on the systematic and comprehensive training of its professional knowledge and operation skills. Through modulation and small class teaching, on the one hand, it can help students to modulus and systematize the course of Pharmaceutical Analysis, improve the flexible application ability, understand the working principle of the module, and use it. Maintenance of equipment, on the other hand, can solve the actual problems of drug production, from the perspective of the establishment of students' knowledge system and the practical application of analysis and detection methods, to achieve the purpose of skilled application.

This specialty trains personnel mainly to be engaged in drug development, production, storage, sales, use of quality testing and drug quality management technology application-oriented professionals. The specialty is developed on the basis of the "Pharmaceutical quality testing technology" specialty, which focuses on the quality control in the pharmaceutical production process. [15] This specialty trains relevant theories and operational skills of drug quality and safety, possesses good professional ethics and professional accomplishment, and engages in pharmaceutical production raw materials, intermediates, drug quality inspection, quality control, new drug research and development analysis, drug safety and quality assurance to meet GMP requirements in the pharmaceutical industry. Graduates can effectively use analytical techniques to judge and evaluate the quality and safety of APIs and medicines, abnormal production process, timely and correct guidance of production, to ensure the quality and safety of pharmaceutical analysis and testing of front-line skilled professionals. In addition to organizing students to take computer grade and English grade examinations every year, this major also organizes students to take part in training and certification of drug inspector and analysis grade, encourages students to obtain ISO quality internal auditor certificate, and enables students to achieve zero docking with enterprises after graduation.

\section{2. "Pharmaceutical Analysis" Module Teaching}

The MES module reform based on the supply side of Pharmaceutical Quality \& Safety Speciality "Pharmaceutical Analysis" is based on the characteristics of the subject, its status and role in the whole professional system, and the related requirements of the Chinese Pharmacopoeia, and the two parts of the "Pharmaceutical Analysis", namely, the theoretical and experimental articles, and the 20 modules. It is divided into 17 modules according to the theoretical system of Pharmaceutical Analysis, including the identification of common drugs, the routine examination of drug impurities, the determination of the content of drug related substances, the general situation of drugs and drug management, the analysis of pharmaceuticals, the development of new drugs, the nature of the representative drugs and the detection methods. Three modules, of which basic training projects set up 17 training projects for the basic skills training module of the theoretical module, improve the module 5 training projects, and the comprehensive ability training module 5 practical training projects. The module's total time is 160 hours, and the teaching sequence is carried out in the form of theory and practice. [16] The module's total time is 160 hours, and the teaching sequence is carried out in the form of theory and practice. This kind of teaching method first highlights the integrity, based on the inner connection of knowledge points and the students' understanding, and presents the basic skills training and theory in the form of a whole module in front of the students, so that they will no longer face the course in isolation and boring, but integrate all the knowledge points together with the analysis test. Secondly, it can enable students to contact the frontiers of the industry as soon as possible, and help students to explain the basis and origin of each Pharmacopoeia and the national standard method with the basic theory knowledge. This educational idea conforms to the new era of new analysis and inspection talents to a certain extent, this is MES The significance of the modulus teaching reform [5].

\section{Teaching Reform of Pharmaceutical Analysis Module}

\subsection{Teaching Content}

In 1980, one of the founders of pharmaceutical analysis in China, Mr. Andengkui, in his edited Drug Analysis, pointed out that pharmaceutical analysis mainly uses chemical, physical and chemical methods to study the quality of synthetic drugs or natural drugs and their preparations with clear chemical structure. Zeng Su and others put forward that 
pharmaceuticals analysis is to ensure and control the quality of drugs in an all-round way by using physical, chemical, physical, chemical, microbiology, informatics and other methods, through the development, manufacture and clinical use of all aspects of the process. Pharmaceutical analysis includes two major research fields: drug analysis technology and drug analysis science. The former focuses on applied research, while the latter focuses on basic or applied research. Combining the characteristics of the subject of drug analysis with the actual situation of Higher Vocational education, we designed the content of the course of drug analysis modulus.

In contrast to the training program of Pharmaceutical Quality \& Safety Speciality professionals, Pharmacopoeia, the syllabus of the licensed pharmacist and the demand for talents in the industry, the teaching mode of MES modular personnel training is deeply understood and internalized, and the current teaching contents are examined in a modular and systematic way, thus ensuring the smooth content of the content, and the specific organization form is the MES model. Each teaching and research department in the block has a planned and planned organization module for all the instructors to prepare class preparation and trial system. At the same time, the industry experts are invited to form the Specialized Committee to put forward opinions and questions. Teachers "lecture" and collective lesson preparation to review the current teaching content one by one, examine and unify teachers' master of training programs, grasp the key and difficult points, discuss and modify teaching cases and courseware; secondly, break the barriers between the modules and even the modules within the module, all the modules are taught. The lab requires all the young teachers to take specific lectures on the other courses in the module, so as to realize the emphasis on the teaching of other courses or other modules related to the modules. At the same time, ensure that the content of each course is smoothly linked, knowledge points are covered and omissions, so as to optimize the teaching content and achieve the effect of deep integration of modules. For example, the course of drug chemistry and Pharmaceutical Analysis will teach the section of central neurosurgeon, but the course of drug chemistry focuses on chemistry, focusing on the structure, nature, principles, processes and uses of drugs, while the course of Pharmaceutical Analysis focuses on structure, nature and principles. The application of the analysis method, in the course of the course of "drug chemistry" before, "Pharmaceutical Analysis" in the post, to ensure that the content smooth, also in line with the students' cognitive rules; and as: the "Chinese Pharmacopoeia" and use, in the "Pharmaceutical Analysis" and "Pharmaceutical Analysis comprehensive training" as a module, the former let the study Students understand and understand the basic functions of the Pharmacopoeia. The latter is further applied on the basis of proficiency to make it a complete reference book for students. Through the adjustment of the teaching content, the integration of modules, modules and other courses can be seamless, so as to achieve the true integration of modules and achieve a predetermined teaching goal. The interaction effect of teachers and students is good, the teaching effect has been improved significantly, and the growth of young teachers is also promoted [17].

\subsection{Teaching Organization}

As the lead Department of the MES module, Pharmaceutical Quality \& Safety Speciality teaching and research department can fully play an overall organizational role and coordinate the teaching and research departments of the modules to complete the teaching arrangements and teaching tasks on time. Before the beginning of the class, the other relevant Teaching and Research Department are organized to formulate the timetables in a unified manner. After that, the teaching and research departments shall immediately convene the teaching task arrangement and teaching preparations according to the timetable and the teaching schedule and the teaching tasks assigned by the school, and carefully analyze the situation of teachers and the special points of the teaching objects, and allocate the teaching tasks reasonably. The teaching group was set up, the teaching implementation plan was formulated, and teachers were allowed to make use of the vacation time to carry out the teaching design and teaching preparation in advance. During the course of the course, we insist on the director of the teaching and research room and the subject leader to listen to the class, examine the teacher's classroom teaching, make suggestions on the teaching methods of the teachers, ask the teachers to use various teaching methods, pay attention to the interaction between teachers and students, and activate the atmosphere of the classroom. After the end of the course, the teaching summing up meeting will be held in time to sum up the teaching experience and provide reference for the follow-up teaching work. In addition, each Teaching and Research Department is supervised by the director, and the backbone teachers supervise to ensure seamless connection.

\subsection{The Introduction of PBL Teaching Model}

Problem-Based Learning (PBL) is a typical student-centered teaching method, which places learning in a complex and meaningful problem situation and allows students to solve problems in the process of learning together in groups to learn the scientific knowledge hidden behind the problems. In order to promote their autonomous learning and lifelong learning ability. The real life scene simulation and reproduction will lead students into the scene, the students through the simulation of the role, the full use of knowledge learned for problem analysis and decision-making. PBL teaching method can not only enable students to acquire knowledge in discussion, but also improve their interpersonal communication ability through discussion. Because the teaching cases are true and the materials are rich, it is easier for the students to treat, analyze and solve problems in their own situation [18].

The pharmaceutical analysis module innovator learned from the PBL teaching mode of medical specialties. The PBL teaching mode is a new teaching method which takes students as the center, and takes the problem as the guidance, and gives 
full play to the students' initiative to explore the problems and solve the problems. It is also the focus of the reform of the teaching methods in the teaching reform of the MES module. The PBL teaching mode focuses on 5 training projects, namely, the upgrading module, 5 training projects and the comprehensive ability training module. It lays the foundation for the following course "comprehensive analysis of pharmaceutical analysis" to form students' autonomous learning atmosphere and active experimental ability. In addition, teachers organized lectures and observation on the PBL course of pharmaceutical analysis, and strengthened the training and learning exchange of PBL teachers. In the future, PBL tutor group will be set up in the module, and the unified understanding of PBL teaching will be further improved. Through comparative study, we can see that the new teaching mode has more advantages in higher vocational teaching. It can fully arouse enthusiastically for learning, deepen students' understanding and mastery of knowledge, thus improving learning efficiency. Higher vocational education provides vast space for advanced teaching mode. It is a trend to promote the reform of teaching mode through the joint efforts of teachers and students. According to the characteristics of courses, one or several teaching modes should be selected to combine and explore an effective teaching mode.

\subsection{Assessment Methods}

The assessment method has always been the focus of students' attention and the focus of teaching reform. According to the requirements of the curriculum standard and the training of professional talents, the traditional examination paper examination mode has been abandoned and the process assessment has been strengthened. The three parts of the subject examination, including the written examination, the operation and the ordinary performance, are composed of the comprehensive examination $(30 \%)$, the operation $(40 \%)$, and the normal (30\%). The operation examination also includes the basic professional accomplishment, such as the ability to solve the problem, the ability to operate, the ability of teamwork and the communication ability of the members, and highlight the characteristics of the combination of work and Engineering in the vocational colleges, so as to evaluate the students' two-way learning effect on the theory and practice skills [20-21]. On the other hand, in order to adapt to the knowledge update brought by the rapid development of various disciplines, under the unified organization of the school, the Department of research and research of the Pharmacopoeia of the Pharmacopoeia of Pharmacopoeia has been updated in a timely and systematic way to ensure the diversification, non repetition and keeping pace with the times. Through the exploration and practice of recent years, we have found that the diversified assessment methods can not only optimize the assessment scheme, reduce the burden of students, encourage them to participate in the enthusiasm, but also can reflect the students' learning effect more comprehensively and truly, and evaluate the study effect of their theory and practice, which is worth continuing to carry out.

\section{Problems and Thinking in the Reform of Module Teaching}

The implementation of MES module teaching is a brand-new attempt, reflecting the determination and courage of this school's reform of education mode. From the beginning of the reform of the "Pharmaceutical Analysis" MES module in 2014, the concrete implementer of the reform, including all the teachers and students, have paid their own efforts, but the problems still exist.

\subsection{Top Floor Design Needs to Be Perfected}

The object of education is the students. The ultimate aim of the teaching reform is to adapt to the requirements of the times from the perspective of the supply side and to train the Pharmaceutical Analysis and testing personnel to adapt to the requirements of the development of the new era. Therefore, in the course of the reform of the course MES, students should be taken as the main body and all the students are set out as the starting point. The reform has put forward higher requirements for the quality of teachers. Teachers should have the ability of professional system and the ability to control the whole world. After the reform, the curriculum also makes more knowledge have relevance. The system framework of the whole course is becoming more and more complex, which seems to bring more challenges to students' study and how to be the whole. It is a problem that the next step of the course design needs to be solved. [22]

\subsection{The MES Reform Programme Needs to Be Further Optimized and Deepened}

The current MES reform is still in its infancy, and the convergence of individual content needs to be optimized without reaching a true depth of integration. "Pharmaceutical Analysis" has the characteristics of both theory and practice. How to combine the boring theory with the more interesting operation of the students will be the key and difficult point of the next reform. MES teaching reform must combine practice with theory reasonably, strengthen practice teaching and achieve three changes. One is the transformation from teacher centered education mode to student centered teaching mode. The two is to transform knowledge into skill training. The three is the shift from teaching oriented classroom to simulated production practice.

\subsection{Training for Teachers Is Also Facing Great Challenges}

Some of the teachers of the MES reform team are graduate of analytical chemistry, or although they are graduate of pharmacy, they are lacking in the work experience of the enterprise, and are not very close to the frontiers of the development of pharmaceutical analysis in the industry. These objective problems have restricted the further advance of the curriculum reform of the course MES. But in the solution of this problem, our hospital has made a meaningful attempt. We require all teachers with Double Teachers' qualifications to take 6 months' time in a part-time job in the enterprise within 5 
years of the teaching practice, which makes the teachers have more opportunities to contact the first line of analysis and unknowingly update them. The teacher's knowledge of Pharmaceutical Analysis. In addition, the medicine quality and safety teaching and research department also invites professional lectures of industry experts. It does not take up the basic theory teaching time, but also opens the students' thinking of Pharmaceutical Analysis, so that students can contact the thinking mode of Pharmaceutical Analysis in advance. These measures are of great significance to the further development of MES reform [23, 24].

\subsection{The Quality of PBL Teaching Needs to Be Improved}

PBL teaching is quite different from previous teaching methods in terms of teacher teaching and student learning, so there are still many problems. Teachers are quoted in cases, inspiring students' ideas, encouraging students to speak not enough, teaching ideas still deviant, some teachers' organization in class discussion is not high, and lack of analysis and thinking. The concept, process and organization of PBL teaching for individual teachers are not familiar enough, and training and learning exchanges need to be strengthened. Through comparative study, we can see that the new teaching mode has more advantages in higher vocational teaching. It can fully arouse enthusiastically for learning, deepen students' understanding and mastery of knowledge, thus improving learning efficiency. Higher vocational education provides vast space for advanced teaching mode. It is a trend to promote the reform of teaching mode through the joint efforts of teachers and students. According to the characteristics of courses, one or several teaching modes should be selected to combine and explore an effective teaching mode.

\section{Conclusion}

To sum up, "Pharmaceutical Analysis" as the core course of pharmacy and related major, we combine the situation of Pharmaceutical Quality \& Safety Speciality students, try to carry out the MES module reform. The reform takes the historical facts of Pharmaceutical Analysis and development, guides the students' interest and the characteristics of pharmacology professionally, rationally chooses the contents of the course, introduces the frontiers of the application and research of Pharmaceutical Analysis, and directs the drug food safety, and makes clear the importance of Pharmaceutical Analysis knowledge to ensure the safety of drug use. The reform takes the drug food safety as the guidance, clear the importance of the Pharmaceutical Analysis knowledge to ensure the safety of drug use, based on the typical Pharmacopoeia analysis method, learning the new technology of Pharmaceutical Analysis experiment in the use of drug testing, paying attention to the teaching methods and methods, transforming the abstract into inquisition and reducing the difficulty of the theory of the theory. The reform measures of Pharmaceutical Analysis MES enlighten students' interest in learning better, and make students realize that drug quality testing is a guarantee subject closely related to the healthy development of the pharmaceutical industry; the introduction of the research frontier of Pharmaceutical Analysis and the introduction of typical methods and techniques makes students in the actual work in the future and develop drug quality inspection, the new idea of measuring technology.

\section{Acknowledgements}

*Chongqing foundation and frontier research program (No:cstc2016jcyjA0075) Chongqing higher education reform research project (No:143184); Chongqing Chemical Industry Vocational College (CCIVC), HZY2016-jy03 project funding.

\section{References}

[1] Wang Jincheng, Guo Yan. Exploration and reform of "multi module and three dimensional" practical teaching system. Polymer bulletin, 2012, (10). 90-94.

[2] Qian Chunrong, Cheng Hongying, Yang Yanni. Modularization reform and application of basic nursing courses. Chinese nursing management. 2015. 0 (7). 828-831.

[3] Song Lili, Bao Yuli, Liu Hongyu. Reform of Comprehensive Design Experiment Based on Pharmaceutical Analysis Experiment Course. Laboratory Research and Exploration, 2016, 35 (6); 185-189.

[4] Ouyang Hui. Exploration on experimental teaching of pharmaceutical analysis. Occupation, 2016, 0 (14).91.

[5] Li Xiaojuan, Guo Yake, Zhu Shifa et al. Experimental Teaching Reform of Pharmaceutical Analysis Based on Cultivation of Practical Ability. Guangzhou Chemical Industry, 2017, 45(4); 128-129.

[6] Ji Xi, Zhou Haipei, Zou Yunfei et al. Setting up and thinking of integrated experiment of drug analysis. Communication of chemical design. 2017. 43 (10). 180-180.

[7] Xiong Yan, Bi Dongqin, Cen Fabo. Reform and Practice of Modular Teaching of Pharmaceutical Analysis under the Training Mode of Applied Talents. Modern Vocational Education, 2016, 0 (27). 34-35.

[8] Li Baiyu, Zhou Jun, Huang Yanfang. Construction of a Mixed Teaching Model for Pharmaceutical Analysis Technology Course. Journal of Nantong Vocational University, 2017, 31 (4). 53-56.

[9] Zhang Liying, Xu Guanhong, Du Shuhu. Thinking and Exploration on the Teaching of Pharmaceutical Analysis in Clinical Pharmacy. Basic Medical Education, 2017, 19 (12). 953-955.

[10] Li Yingchun, Wu Bingbing, Feng Rong. Practice of Reforming Pharmaceutical Analysis Teaching from the American Educational Model. Health Vocational Education, 2014, 32 (18). 34-35.

[11] Chen Cheng, Cao Yin, and Tong Menglan. Research on the Construction of Practical Teaching Model Based on Post Vocational Competence: Taking Pharmaceutical Analysis Course as an Example. Guangxi Education, 2017, 0(2). 22-24. 
[12] Fu Chunmei, Qian Guangsheng. Practice of Inquiry Teaching in Pharmaceutical Analysis Experiment Teaching. Pharmaceutical Education, 2016, 32 (5).52-54.

[13] Wu Lingfeng, Xie Huasong, Luo Baoping. Application of PBL teaching mode in pharmaceutical analysis teaching of clinical pharmacy. Guangdong Chemical Industry, 2017, 44 (13). 315-315.

[14] Ma Ning, Xu Fang, Wang Jianfeng et al. Probe into the application of PBL teaching method in the teaching of drug analysis. Chinese Medical Bulletin, 2010, 7 (31).109-110.

[15] Guan Zhengqiang, Zhu Xincai, Li Wenhua, et al. Exploration and practice of practical teaching mode and operation mechanism [J]. education and occupation, 2008, (24): 140-142.

[16] Tang L, Zou D J, Tang H M. Exploration on the Reform of Multiple Teaching Modes of Pharmacy Based on MES Mode [J]. Education Teaching Forum.2017.

[17] Shi-Tang M A, Xiong Y Y, Zhang X L, et al. Teaching Reform and Practice of Pharmaceutical Analysis Course in Pharmaceutical Major of Applied High Level Universities [J]. Journal of Anhui Science \& Technology University. 2016.

[18] Zhu Q, Ming-Juan L I, Li-Hua L V. Reform and Practice of the Teaching Method of Pharmaceutical Analysis Experiment Course [J]. Guangzhou Chemical Industry, 2016.

[19] Liang Chao, Fang Zhao Qin, Pan Zhiqiang. Preliminary exploration of PBL teaching of experimental Chinese medicine based on curriculum design. Shi Zhen Guo Yao Guo Yao, 2017, 28 (9); 2253-2254.
[20] Guo Zhangyan, Chen Lihua, Wen Weihong. Summary and Reflection on module teaching of immunity and infection basis. Chinese Journal of immunology, 2016, 32 (9); 1374-1376.

[21] Han Z, Ren Y, Zhu J, et al. Multianalysis of 35 Mycotoxins in Traditional Chinese Medicines by Ultra-High-Performance Liquid Chromatography-Tandem Mass Spectrometry Coupled with Accelerated Solvent Extraction [J]. Journal of Agricultural \& Food Chemistry, 2012, 60(33):8233-47.

[22] Liu Wenying. An Deng. Drug analysis. Fifth Edition [M]. people's Health Publishing House, 2003.

[23] Zeng Su, He Langchong, Luo Guoan, et al. [J].Journal of Pharmaceutical Analysis, 2013 (9): 1645-1647.

[24] $\mathrm{Hu} \mathrm{N}, \mathrm{Wu} \mathrm{C}, \mathrm{Ha} \mathrm{D}$, et al. A novel microphysiometer based on high sensitivity LAPS and microfluidic system for cellular metabolism study and rapid drug screening. [J]. Biosensors \& Bioelectronics, 2013, 40(1): 167-173.

\section{Biography}

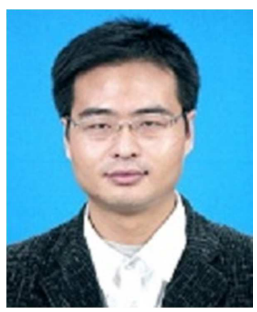

Wang Wenbin (1980--), male, is now working at the Career Academy in Chongqing chemical industry, lecturer, major director of Pharmaceutical Quality \& Safety Speciality: nonlinear chemistry. 